\title{
Electrically detected magnetic resonance in a W-band microwave cavity
}

\author{
V. Lang, ${ }^{1, *}$ C. C. Lo, ${ }^{2, *}$ R. E. George,${ }^{3}$ S. A. Lyon, ${ }^{4}$ J. \\ Bokor, ${ }^{2}$ T. Schenkel, ${ }^{5}$ A. Ardavan, ${ }^{3}$ and J. J. L. Morton ${ }^{1,3}$ \\ ${ }^{1}$ Department of Materials, University of Oxford, \\ Oxford OX1 3PH, United Kingdom \\ ${ }^{2}$ Department of Electrical Engineering and Computer Sciences, \\ University of California, Berkeley CA 94720, USA \\ ${ }^{3}$ CAESR, Clarendon Laboratory, Department of Physics, \\ University of Oxford, Oxford OX1 3PU, United Kingdom \\ ${ }^{4}$ Department of Electrical Engineering, \\ Princeton University, Princeton NJ 08544, USA \\ ${ }^{5}$ Accelerator and Fusion Research Division, \\ Lawrence Berkeley National Laboratory, Berkeley CA 94720, USA
}

(Dated: January 24, 2011)

\begin{abstract}
We describe a low-temperature sample probe for the electrical detection of magnetic resonance in a resonant $\mathrm{W}$-band $(94 \mathrm{GHz})$ microwave cavity. The advantages of this approach are demonstrated by experiments on silicon field-effect transistors. A comparison with conventional low-frequency measurements at X-band $(9.7 \mathrm{GHz})$ on the same devices reveals an up to 100-fold enhancement of the signal intensity. In addition, resonance lines that are unresolved at X-band are clearly separated in the W-band measurements. Electrically detected magnetic resonance at high magnetic fields and high microwave frequencies is therefore a very sensitive technique for studying electron spins with an enhanced spectral resolution and sensitivity.
\end{abstract}

\footnotetext{
* Both authors contributed equally to this work. Please contact the corresponding author under volker.lang@materials.ox.ac.uk.
} 
Electron paramagnetic resonance [1] (EPR) is an important spectroscopic technique used to identify and study electron spins in various material systems. Most conventional EPR measurements are carried out at a microwave frequency of around $9.7 \mathrm{GHz}$ (X-band). For material systems with an electron gyromagnetic ratio of $g \sim 2$, this corresponds to a resonant field of approximately $0.35 \mathrm{~T}$ and a Zeeman splitting of $40 \mu \mathrm{eV}$. Hence, the sensitivity of conventional X-band EPR is limited and typically requires at least $10^{10}$ paramagnetic sites in the sample [2]. In order to enhance the detection sensitivity, EPR has been combined with optical [3,4] and electrical detection schemes [5-8]. Electrically detected magnetic resonance (EDMR), in which the EPR induced change in spin population is detected through the change in conductivity of the sample $[12,13]$, has been reported to provide a more than $10^{6}$ times higher sensitivity than conventional EPR [9-11].

Higher EPR sensitivities and spectral resolutions can be achieved by using higher magnetic fields and higher microwave frequencies, as the EPR signal intensity scales with the spin polarisation, and the $g$-factor resolution with the operating frequency, linearly [14]. High sensitivities and high spectral resolutions are both very desirable and of great importance for the characterisation of material systems, such as defect identifications in solar cells, and the emerging fields of spintronics and quantum computation, as they offer the opportunity to selectively address and study single electron spins. Furthermore, a comparison of the same device at different magnetic fields provides information on the microscopic mechanism that gives rise to the EDMR signal.

So far, high-field EDMR has been carried out at $8.50 \mathrm{~T}$ on Si:P [15-17] with a multimode (Fabry-Pérot) cavity only, which requires comparatively long pulse duration times of the order of a few hundred nanoseconds for a $\pi$-pulse $[15,16]$. The sensitivity in those experiments was limited to $\sim 5 \times 10^{7}$ spins in the sample [15]. The implementation of fast microwave pulses with large excitation bandwidths for the coherent control of electron spins via pulsed-EDMR [18] thus remains technologically challenging and has not been achieved at high magnetic fields so far. Single-mode cavities on the other hand offer fast microwave pulses ( $\pi \sim 32 \mathrm{~ns}$ in our system) to achieve large excitation bandwidths. In addition, the distribution of the microwave field in single-mode cavities is well defined. Hence, the sample can be placed at the position where the magnetic field component is maximal and 
the electrical field component minimal, which is much more difficult to achieve in multimode resonators and essential for the minimisation of microwave-induced rectification noise. This work combines the advantages of single-mode cavities with the enhanced sensitivity of an electrical readout and the enhanced spectral resolution at high microwave frequencies and high magnetic fields. In particular, we describe our approach to perform EDMR in a single-mode microwave cavity at $3.36 \mathrm{~T}$ and $94 \mathrm{GHz}$ (W-band).

(a)

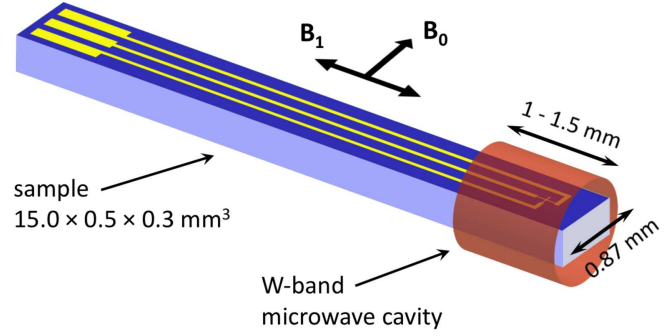

(b)

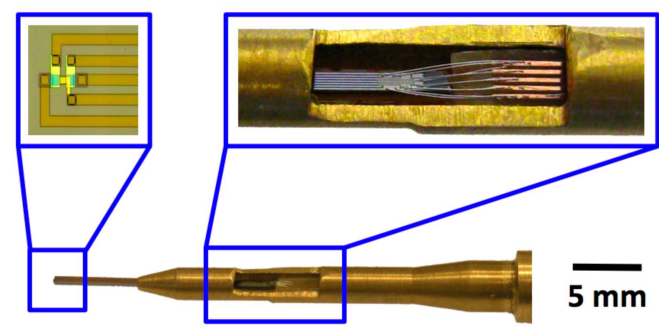

FIG. 1. (colour online). (a) Schematic drawing showing the sample placement inside the W-band EPR microwave cavity. Black arrows indicate the orientations of the static magnetic Zeeman and microwave fields, $B_{0}$ and $B_{1}$, respectively. (b) shows a series of optical images of a sample (topleft) being inserted into the brass collet (bottom) used to guide the device into the cylindrical microwave cavity. Aluminium wirebonds connect the device with a printed circuit board and are visible through the window in the sample collet (top-right).

Since the half-wavelength of free space microwaves at $94 \mathrm{GHz}$ amounts to $1.6 \mathrm{~mm}$, the size of a W-band microwave resonator typically lies within the range of a millimetre and EDMR experiments require (i) a small sample size, (ii) an optimised geometry and metallisation for reduced microwave absorption, and (iii) an accurate sample placement inside the resonator. The sample probe wiring has to enable the detection of small voltages and currents with a low noise level. Our low-temperature sample probe was designed for use in a commercial Bruker Teraflex EN 600-1021H TE 011 mode pulsed electron nuclear double resonance (ENDOR) resonator, which has an inner diameter of $0.87 \mathrm{~mm}$ and a length of 
$1-1.6 \mathrm{~mm}$, depending on the particular tuning condition. The sample placement inside the W-band microwave cavity as well as the most important dimensions are shown in Fig. 1 (a) schematically. The sample collet developed for this work facilitates a safe and accurate sample placement inside the microwave cavity and is shown in Fig. 1 (b). It consists of a brass tip, which allows accurate centering of the device inside the cylindrical cavity along the symmetry axis. A micrometer screw equipped with a vacuum seal at the probe head is used to withdraw the fragile sample within the collet in order to protect it while the probe is inserted into the cryostat. Once the sample probe is aligned against the resonator window, the micromanipulator can be used to push the sample out of the collet and to position it inside the resonator with $\sim 100 \mu \mathrm{m}$ precision. Withdrawing the sample from the cavity back into the collet protects the device during the extraction of the sample probe from the cryostat once the experiment is finished. We have also machined a sample collet out of G1040 glass epoxy, with which the same sample can be measured in a conventional X-band system without disturbing the microwave field and without changing the sample probe. The sample is connected to a double-sided printed circuit (PC) board via aluminium wirebonds, which are visible through a window in the brass tip as shown in the top-right image of Fig. 1 (b). This arrangement allows us to contact the active area of the device by metallic layers with a thickness of $\sim 0.1 \mu \mathrm{m}$ below the skin depth of microwaves at $94 \mathrm{GHz}$ [14] and to minimise the disturbance of the microwave field in the resonator. The PC board is equipped with a 6-pin Omnetics PZN-06-DD miniature connector at its top end, which is connected to stainless steel coaxial cables (LakeShore CC-SS) [19]. The coaxial lines terminate in female vacuum-tight SMA connectors at the probe head. From the capacitance of the coaxial cables used in the probe stick $(\sim 200 \mathrm{pF})$, we expect a measurement bandwidth of $10 \mathrm{kHz}$ to be possible with the current set-up for a sample resistance of $\sim 100 \mathrm{k} \Omega$.

We have used this sample probe to detect the EDMR effect of conduction band electrons in silicon-based n-type accumulation-mode field-effect transistors (aFETs). The results presented in this work are representative for all measurements. The device has a triple-gate geometry for enhancing the spin polarisation of the injected conduction electrons, but for the purpose of this study, all three gates are biased together and the whole device is considered as a simple three-terminal aFET. The device has a total channel length of $160 \mu \mathrm{m}$ and width $40 \mu \mathrm{m}$, and is fabricated on a silicon substrate with a $1 \mu \mathrm{m}$ thick isotopically 
purified ${ }^{28} \mathrm{Si}$ epitaxial layer with $3 \times 10^{16} \mathrm{~cm}^{-3}$ phosphorus doping. In addition, the channel was implanted with arsenic $\left({ }^{75} \mathrm{As}\right)$ donors at $50 \mathrm{keV}$ and a dose of $4 \times 10^{11} \mathrm{~cm}^{-2}$. From secondary ion mass spectroscopy (SIMS) we estimate approximately $6 \times 10^{5}$ arsenic and $4 \times 10^{6}$ phosphorus donors residing within $10 \mathrm{~nm}$ of the oxide interface of the device channel. The device layout is optimised for microwave cavity-based EDMR measurements with minimal metal thicknesses. All samples were diced into small chips of $15.0 \times 0.5 \times 0.3 \mathrm{~mm}^{3}$ in size, and connected to the sample probe with the sample arrangements described above.

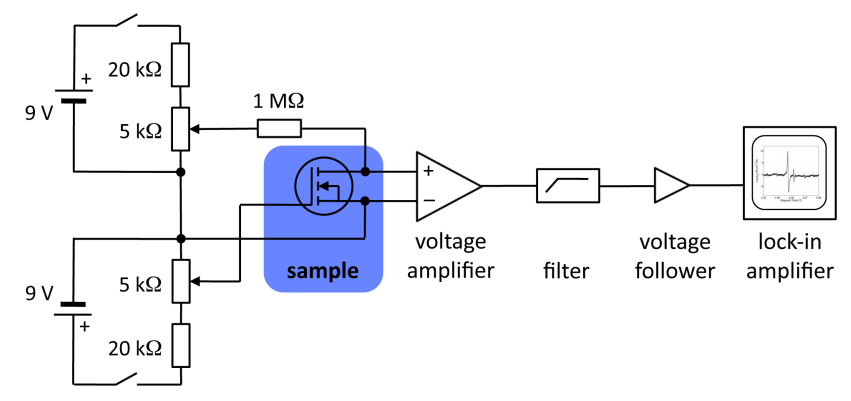

FIG. 2. (colour online). Circuit diagram of the electrical measurement setup used to detect EDMR on silicon field-effect transistors. Please refer to the text for further details.

W-band EDMR was performed with a Bruker ElexSys E680 W-band EPR spectrometer and an Oxford Instruments CF-1200 helium-gas flow cryostat in combination with an Oxford Instruments ITC-503S temperature controller. The DC magnetic field $B_{0} \sim 3.36 \mathrm{~T}$ was generated with a Bruker EPR 6T SC superconducting magnet. X-band EDMR measurements were carried out on the same device with a Bruker ElexSys E680 X-band EPR spectrometer and an Oxford Instruments CF-935 helium-gas flow cryostat. The microwave excitation was applied using a Bruker Flexline ER 4118X-MD5-W1 X-band dielectric ring resonator operating at a DC magnetic field $B_{0} \sim 0.35 \mathrm{~T}$. All measurements presented in this work were carried out at $T=5 \mathrm{~K}$, and the Zeeman field was aligned in the plane of the 2DEG, perpendicular to the direction of current flow. Magnetic field modulation was used at both microwave frequencies to enhance the signal-to-noise ratio, which results in the EDMR signal appearing as the first derivative of the sample resistivity with respect to magnetic field, i.e. $\partial\left(\Delta \rho / \rho_{0}\right) / \partial B$. The circuitry used to provide isolation from mains noise and to detect the resonant change of the sample resistivity is shown in Fig. 2. Battery powered variable resistor networks were used to apply a constant current to the sample and to bias it with a constant gate voltage, typically $300 \mathrm{mV}$. The resonant change of the source-drain 
voltage across the aFET was detected via a battery powered variable gain low-noise voltage amplifier (Femto DLPVA-100-F-D) and a bandpass filter (Princeton Applied Research 5210 lock-in amplifier) tuned to the modulation frequency of $5.02 \mathrm{kHz}$. A voltage follower (OPA TL074CN) facilitated the impedance matching between the whole circuitry and the software driven lock-in amplifier of the Bruker ElexSys E680 unit, which was used to demodulate and detect the EDMR signal. We used a field modulation amplitude of $0.2 \mathrm{mT}$ throughout, calibrated at both W- and X-band.

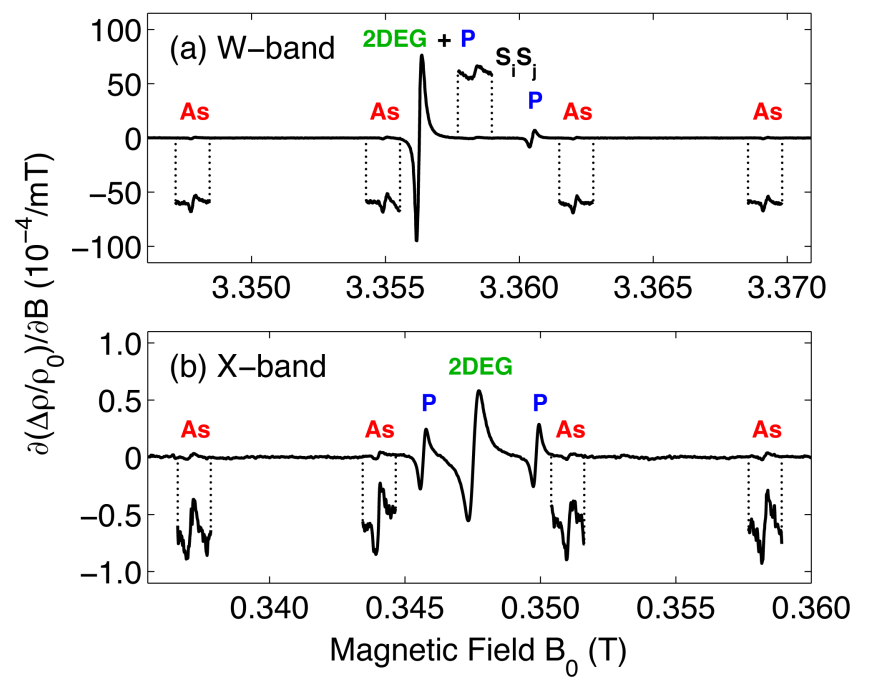

FIG. 3. (colour online). Typical W- (a) and X-band EDMR spectra (b). The main resonance is due to the $2 \mathrm{DEG}$, the weaker lines due to ${ }^{31} \mathrm{P}$ and ${ }^{75} \mathrm{As}$ donors, respectively. The resonance line associated with exchange-coupled donor pairs and clusters $S_{i} S_{j}$ is well separated from the 2DEG at W-band only. 10-fold zooms are shown for the exchange-coupled donor pairs and ${ }^{75}$ As lines and offset for clarity.

The W- and X-band EDMR spectra of a typical aFET are shown in Fig. 3. In both spectra, the resonance line with the largest amplitude has a $g$-factor of $g=1.9999$ and corresponds to the two-dimensional electron gas (2DEG) [20, 21]. The two satellite peaks of the ${ }^{31} \mathrm{P}$ donors are evidenced by the expected phosphorus hyperfine splitting of $4.2 \mathrm{mT}$ [22]. Four weaker resonances are separated by $7.1 \mathrm{mT}$ from each other, which corresponds to the four different nuclear spin projections of ${ }^{75}$ As donors [23]. All donor lines are centered around $g=1.9987$, and hence the low-field hyperfine-split resonance of the ${ }^{31} \mathrm{P}$ donors is superimposed with the large 2DEG resonance in W-band as the difference in $g$-factors corresponds to $-2.1 \mathrm{mT}$ at that frequency. Exchange-coupled donor pairs and clusters give 
rise to a resonance signal $S_{i} S_{j}$ at the center of the hyperfine-split lines [24], which overlaps with the 2DEG resonance at X-band, and is well separated from the large 2DEG signal at W-band. This demonstrates the superior spectral resolution in W- over X-band EDMR measurements.

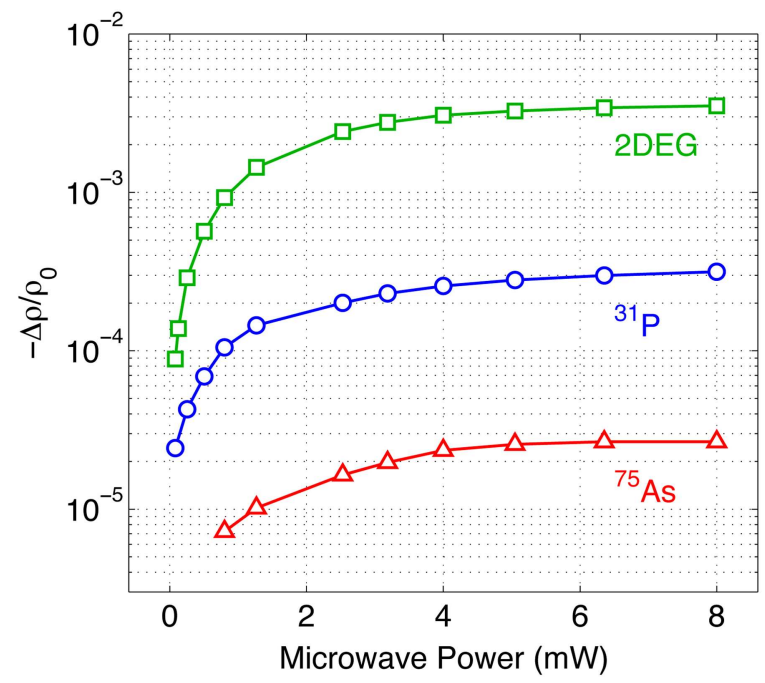

FIG. 4. (colour online). W-band power dependence of the EDMR signal intensity of the 2DEG $(\square)$, phosphorus $(\bigcirc)$, and arsenic $(\triangle)$ line on a semilogarithmic scale. All three lines are well saturated for a microwave power larger than $\sim 5 \mathrm{~mW}$. Solid lines are guides to the eye for clarity only.

Another difference between the $\mathrm{W}$ - and X-band spectra shown in Fig. 3 concerns the EDMR signal intensities. For a better comparison we calculated the EDMR signal intensity by integration of $\partial\left(\Delta \rho / \rho_{0}\right) / \partial B$ with respect to magnetic field and thus define it as the amplitude of the absorption line. This analysis reveals a $\sim 100$-fold increase in signal intensity for the 2DEG and $\mathrm{a} \sim 20$-fold increase for the ${ }^{31} \mathrm{P}$ and ${ }^{75} \mathrm{As}$ donor resonances. Details of this scaling as well as the particular EDMR mechanisms giving rise to these signals are discussed elsewhere [25]. The superior signal amplitudes at W-over X-band improve the signal-to-noise ratio $S / N$ from $S / N=4: 1$ at $\mathrm{X}$ - to $8: 1$ at W-band for a single scan. The number of arsenic spins detected in our experiments is smaller than $\sim 10^{6}$, and from the $S / N$ in our W-band measurements, a sensitivity of $2 \times 10^{4}$ per hyperfine line should be achievable in a single scan. This is very encouraging, particularly since high-field EDMR has not been demonstrated with fewer than $\sim 10^{9}$ spins and a sensitivity better than $5 \times 10^{7}$ so far [15]. 
Fig. 4 shows the microwave power dependence of the EDMR signal intensity of the 2DEG, phosphorus, and arsenic line. It reveals a typical saturation behaviour for all three resonance lines associated with the 2DEG, phosphorus, and arsenic spin system, respectively. The signal intensity of each line reaches its maximum value at around $\sim 5 \mathrm{~mW}$, and the three spin systems are well saturated above this value. Power saturation only occurs if the incident microwave power is high enough to populate the excited spin state of the particular spin system faster than it can relax to its equilibrium population through intrinsic spin-lattice relaxation processes [14]. Our measurements demonstrate that the microwave field $B_{1}$ in our cavity is high enough to induce power saturation of all three spin systems, illustrating another advantage of a resonant cavity approach.

In summary, we have shown that our sample probe can be used to perform EDMR in a single-mode microwave cavity at $3.36 \mathrm{~T}$ and $94 \mathrm{GHz}$. Advantages of this technique were demonstrated and discussed by comparing W- and X-band EDMR spectra of accumulationmode field-effect transistors. This analysis demonstrates that W-band EDMR is a very sensitive technique for the investigation of defect and donor spins in various micro- and nanostructures offering an enhanced sensitivity and a 10 times higher spectral resolution than conventional X-band EDMR.

\section{ACKNOWLEDGMENTS}

The authors thank Alexei Tyryshkin for fruitful discussions and acknowledge funding from EPSRC through CAESR EP/D048559/1, and US National Security Agency under contract number 100000080295. Work at LBNL was supported by the Department of Energy under contract No. DE-AC02-05CH11231. V.L. is supported by Konrad-Adenauer-Stiftung e.V. and EPSRC DTA. A.A. and J.J.L.M. are supported by The Royal Society, J.J.L.M. from St. John's College Oxford.

[1] E. Zavoisky, J. Phys. USSR 9, 211 (1945) 
[2] D. C. Maier, Bruker Rep. 144, 13 (1997)

[3] J. Wrachtrup, C. von Borczyskowski, J. Bernard, M. Orritt, and R. Brown, Nature 363, 244 (1993)

[4] F. Jelezko, T. Gaebel, I. Popa, A. Gruber, and J. Wrachtrup, Phys. Rev. Lett. 92, 076401 (2004)

[5] J. M. Elzerman, R. Hanson, L. H. W. van Beveren, B. Witkamp, L. M. K. Vandersypen, and L. P. Kouwenhoven, Nature 430, 431 (2004)

[6] M. Xiao, I. Martin, E. Yablonovitch, and H. W. Jiang, Nature 430, 435 (2004)

[7] A. R. Stegner, C. Boehme, H. Huebl, M. Stutzmann, K. Lips, and M. S. Brandt, Nature Phys. 2, $835(2006)$

[8] C. C. Lo, J. Bokor, T. Schenkel, J. He, A. M. Tyryshkin, and S. A. Lyon, Appl. Phys. Lett. 91, $242106(2007)$

[9] H. Huebl, R. P. Starrett, D. R. McCamey, A. J. Ferguson, and L. H. Willems van Beveren, Rev. Sci. Instrum. 80, 114705 (2009)

[10] D. R. McCamey, J. van Tol, G. W. Morley, and C. Boehme, Phys. Rev. Lett. 102, 027601 (2009)

[11] C. Boehme and K. Lips, Physica B 376-377, 930 (2006)

[12] J. Schmidt, and I. Solomon, Compt. Rend. 263, 169 (1966)

[13] D. J. Lepine, Phys. Rev. B 6, 436 (1972)

[14] C. P. Poole Jr., and H. A. Farach, Theory of Magnetic Resonance (Wiley, New York 1986)

[15] G. W. Morley, D. R. McCamey, H. A. Seipel, L.-C. Brunel, J. van Tol, and C. Boehme, Phys. Rev. Lett. 101, 207602 (2008)

[16] D. R. McCamey, G. W. Morley, H. A. Seipel, L.-C. Brunel, J. van Tol, and C. Boehme, Phys. Rev. B 78, 045303 (2008)

[17] G. W. Morley, L.-C. Brunel, and J. van Tol, Rev. Sci. Instrum. 79, 064703 (2008)

[18] C. Boehme and K. Lips, Phys. Rev. B 68, 245105 (2003)

[19] Its overall length was chosen such that an additional module built with surface mount components can be attached to the device if necessary.

[20] C. F. O. Graeff, M. S. Brandt, M. Stutzmann, M. Holzmann, G. Abstreiter, and F. Schäffler, Phys. Rev. B 59, 13242 (1999)

[21] S. Shankar, A. M. Tyryshkin, S. Avasthi, and S. A. Lyon, Physica E 40, 1659 (2007) 
[22] G. Feher, Phys. Rev. 114, 1219 (1959)

[23] J. Dirksmeyer, and O. F. Schirmer, Thin Solid Films 184, 55 (1990)

[24] D. New, and T. G. Castner, Phys. Rev. B 29, 2077 (1984)

[25] C. C. Lo, V. Lang, R. E. George, J. J. L. Morton, A. M. Tyryshkin, S. A. Lyon, J. Bokor, and T. Schenkel, e-print arXiv:1012.3811 [cond-mat.mes-hall] 


\section{DISCLAIMER}

This document was prepared as an account of work sponsored by the United States Government. While this document is believed to contain correct information, neither the United States Government nor any agency thereof, nor the Regents of the University of California, nor any of their employees, makes any warranty, express or implied, or assumes any legal responsibility for the accuracy, completeness, or usefulness of any information, apparatus, product, or process disclosed, or represents that its use would not infringe privately owned rights. Reference herein to any specific commercial product, process, or service by its trade name, trademark, manufacturer, or otherwise, does not necessarily constitute or imply its endorsement, recommendation, or favoring by the United States Government or any agency thereof, or the Regents of the University of California. The views and opinions of authors expressed herein do not necessarily state or reflect those of the United States Government or any agency thereof or the Regents of the University of California. 